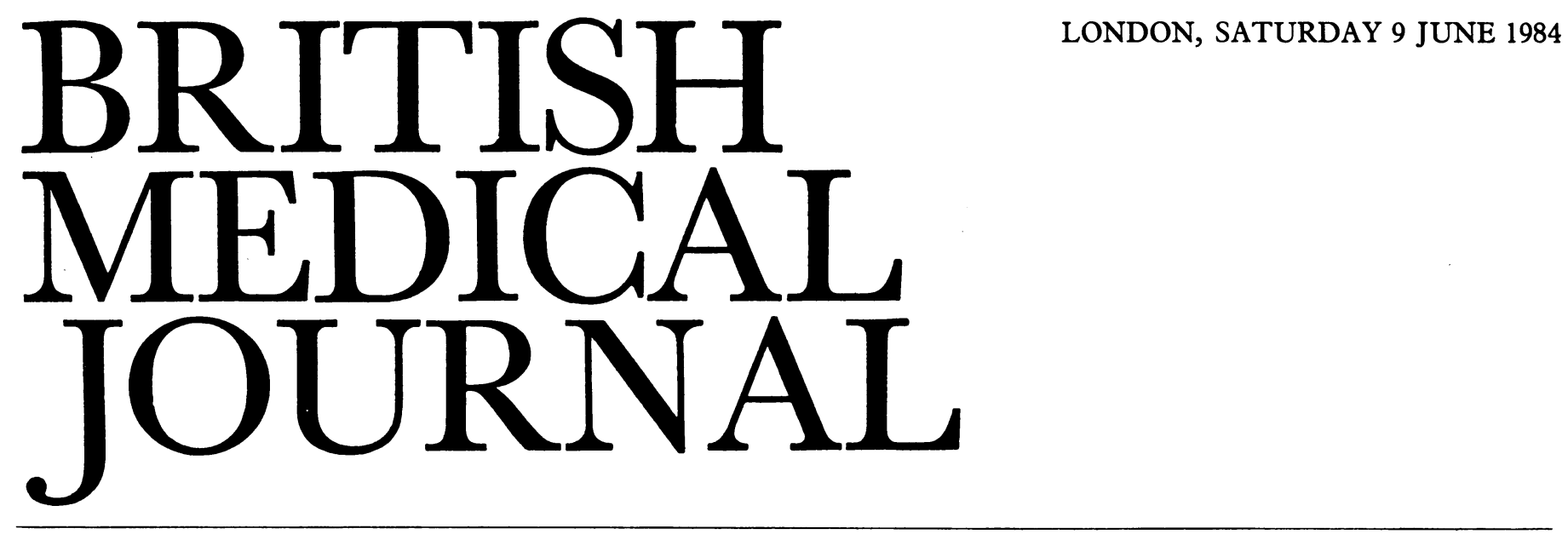

\title{
Adjuvant questions
}

Treatment fails to "cure" breast cancer in a high proportion of patients because the malignant cells disseminate throughout the body when the primary growth is small, and, though treatment for local and regional disease is effective using surgery and radiotherapy, there is no curative treatment for metastatic disease-and it is metastatic disease which kills. Long term follow up studies show that even in so called "early" disease $70 \%$ of patients will sooner or later die of breast cancer, their deaths being related to their metastatic disease. ${ }^{12}$

Over the past 15 years hopes have been raised that early metastatic disease would yield to treatment with cytotoxic drugs and with hormones. These treatments are known to produce remissions in advanced disease and so might be expected to be effective against micrometastases, when the body tumour burden is less. Numerous clinical trials of chemotherapy, hormone therapy, and immunotherapy have been and are still being conducted. In these trials the drugs and hormones are used immediately after the primary treatmentadjuvant therapy-with the intention of eliminating micrometastases, which are subclinical but which are known from experience to be there.

The results of the trials reported so far are confusing and conflicting. ${ }^{34}$ Most claim a prolongation of "disease free interval"- that is, the time between the treatment of the primary tumour and the development of clinically detectable metastases. Some patients who have had adjuvant therapy seem to be surviving longer, but breast cancer is often a slow disease, and many more years must elapse before any survival benefit can be certain - and the studies will need to be repeated. Other well conducted trials have failed to show any improvement in patient survival. The only constant finding is that the chemotherapy has toxic side effects.

If adjuvant chemotherapy was harmless there would be no problem: all patients with breast cancer could have chemotherapy routinely as a part of their primary treatment. Unfortunately, most effective chemotherapy is extremely toxic and produces unpleasant and distressing side effects. Clinicians need to be sure when prescribing such treatment that a patient with "early" breast cancer has micrometastases which need treatment and, if this seems likely, that the possible benefits of chemotherapy outweigh the risks and disadvantages. Many regimens currently used spread toxic treatment over six months, and this can be justified only if it will make a substantial contribution to a patient's future health-and at present this cannot be guaranteed.

C BRITISH MEDICAL JOURNAL 1984. All reproduction rights reserved.
Adjuvant hormone treatment, particularly with the antioestrogen tamoxifen, looks more promising. Trials have shown a prolongation of disease free interval, and an early finding has been an improvement in survival when it is used as an adjuvant treatment. ${ }^{5}$ The bonus with tamoxifen is the lack of toxicity and side effects, but not all patients have hormone sensitive tumours, and there is no sure and simple test for selecting responders.

A clinical trial recently reported from the Institut GustaveRoussy using polyadenylic-polyuridylic acid as an immune stimulator has shown promising results in patients with up to three diseased axillary lymph nodes. ${ }^{6}$ This treatment also appears to be non-toxic and requires further investigation.

With treatment so difficult and uncertain a reliable prognostic index may be extremely helpful. Patients with a good prognosis may then be spared systemic adjuvant treatment, and those with a poor outlook may be selected for adjuvant prophylactic treatment with the hope of future benefit.

The factors which influence prognosis have been carefully studied, and by using all the available information reasonably accurate forecasts can be made which are of practical value in determining treatment policies. Fisher et al have recently published a paper relating prognosis to the number of axillary nodes affected by the disease. ${ }^{7}$ This requires a full axillary dissection and a full pathological analysis. Blamey and his colleagues have developed a prognostic index from a statistical analysis of eight different factors. ${ }^{8}$ The three which were found to be of most value were node stage, tumour size, and pathological grade. A group from the Ludwig Institute for Cancer Research and the Royal Marsden Hospital Research Institute have recently reported the detection of micrometastases using an immunocytochemical method to screen smears of bone marrow taken at primary operation. ${ }^{9}$ Tumour cells were detected in $28 \%$ of patients.

Such studies are of great importance, for they indicate the patients who are likely to have subclinical metastases and who need systemic treatment in addition to local and regional treatments. Some of the tests described are technically difficult and at present are being performed only in specialist centres, but most are well within the scope of surgeons and pathologists in general hospitals. Nevertheless, clinicians will need to be sure that the tests are worth while in terms of their contribution to management.

There is no consensus on how patients with "early" breast cancer should be treated and no consensus on when adjuvant 
hormone therapy or chemotherapy should be prescribed. Many clinicians, particularly those in North America, recommend prophylactic chemotherapy routinely for all patients with any spread to the axillary nodes-and they may be deemed negligent not to do so. Doubts are now being expressed about this policy, however, and the more conservative approach adopted in Britain is gaining ground.

The problem that clinicians have to face is that adjuvant chemotherapy has not been proved to "cure" patients with micrometastases. In some patients it may be important to prolong the disease free interval, but in others systemic treatment may better be reserved for the time when the metastases cause symptoms. Prognostic indicators are only a guide. Some patients with a poor index live out a normal life span and never develop clinically apparent secondary disease. Meanwhile the hope for the future is that with better drugsand perhaps better ways of using the available drugs-adjuvant chemotherapy may improve the results in breast cancer in the same way as the improvement in the outcome of treatment of lymphomas and teratomas.

Clinical research must continue-both to assess new agents and to optimise the use of those currently available. Further research on the biological characteristics of breast cancer with its metastasing potential should help our understanding of the disease, and if markers and simple detectors of hormone sensitivity can be found there should be great strides in the development of potentially curative treatment.

At present the use of cytotoxic drugs as adjuvant therapy should be in the hands of clinicians in specialist units, and when possible patients should be included in studies and clinical trials. Non-toxic adjuvant treatment with tamoxifen will undoubtedly be used widely, but research is still needed on the place of this agent used in this way. There is no reliable information on how much is required or for how long it should be given. These questions should be answered by further clinical trials, and the studies on immunotherapy need repeating and developing.

It can only be an advantage to have as much information as possible on a patient with primary breast cancer. The data collected will given an indication of the pattern and extent of the disease and of the course it is likely to take and will be of help in planning a treatment programme, but the place of adjuvant therapy must still be the subject of research. Many questions remain unanswered. Which patients? What type of treatment? For how long?

DiANA BRINKLEY

Consultant Radiotherapist,

King's College Hospital,

London SE5 9RS

${ }^{1}$ Brinkley D, Haybittle JL. The concept of cure in breast cancer. Leeds: Yorkshire Breast Cancer Group, 1980. (Private publication.)

2 Langlands AO, Pocock SJ, Kerr GR, Gore SM. Long term survival of patients with breast cancer. Br Med $\mathcal{F} 1979$;ii:1247-51.

3 Rubens RD, Hayward JL, Knight RK, et al. Controlled trial of adjuvant chemotherapy with melphalan for breast cancer. Lancet $1983 ; \mathrm{i}: 839-43$.

4 Rossi A, Bonnadonna G, Valagussa P, Veronisi U. Multimodal treatmen in operable breast cancer: five year results of the CMF programme. $\mathrm{Br}$ Med F 1981 ;282:1427-31.

5 Nolvadex Adjuvant Trial Organisation. Controlled trial of tamoxifen as adjuvant agent in management of early breast cancer. Lancet 1983; 257-61.

- Lacour J, Lacour F, Spira A, et al. Adjuvant treatment with polyadenylicpolyuridylic acid in operable breast cancer: updated results of a randomised trial. $\mathrm{Br}$ Med $\mathcal{F} 1984$;288:589-92.

7 Fisher B, Baker M, Wickerham DL, et al. Relation of number of positive axillary nodes to the prognosis of patients with primary breast cancer. An NSABP update. Cancer 1983;52:1551-7.

${ }^{8}$ Haybittle JL, Blamey RW, Elston CW, et al. A prognostic index in primary breast cancer. $\mathrm{Br} \mathcal{F}$ Cancer 1982;45:361-6.

${ }^{9}$ Redding WH, Coombs RC, Monaghan P, et al. Detection of micrometastases in patients with primary breast cancer. Lancet 1983;ii:1271-3.

\section{Day care for patients with psychiatric disorders}

Last year Vaughn reviewed the "disordered development of day care in psychiatry" and made two criticisms. ${ }^{1}$ Firstly, the planning of psychiatric day hospitals and local authority day centres has been poorly coordinated. They are inequitably distributed around Britain, and they vary greatly in the quality of treatment they provide. Few observers disagree with this. ${ }^{2-4}$ Secondly, though some forms of day care have been available for patients with psychiatric disorders for about 40 years, ${ }^{5-7}$ very little is known about either their efficacy or their efficiency. ${ }^{8}$

The tendency towards reduction in the average length of psychiatric inpatient stay may, it seems, be associated with a corresponding increase in the use of day hospitals ${ }^{9}$; but too little attention has been paid to the problems of the "expanding stage army" of long stay psychiatric day patients. ${ }^{10}$ Indeed, the House of Commons Select Committee on Social Services is now conducting its inquiry into community care with special reference to the adult mentally ill and handicapped.

Outpatient care is apparently more economical of time and personnel and on general grounds is preferred to day care for patients with mild psychiatric disorders who are referred to psychiatrists. ${ }^{11}$ Even for patients with serious or chronic psychiatric disorders, however, several studies have been said to show the general superiority of day hospital care over outpatient and inpatient care. ${ }^{12-24}$ The extent to which day care is associated with improvements in either patients' symptoms or their social state is less clear. Moreover, close inspection of these studies invariably shows up methodological inadequacies. For example, the number of patients tends to be small; often there is selection bias, partial or no randomisation, and little control of important variables such as diagnosis, medication, and treatment between discharge and follow up; day care and inpatient care are often ill defined; outcome measures are not standardised or rated blindly; and too many patients are usually lost during follow up. All these reasons reduce the confidence that can be put on the case favouring day care for patients with severe psychiatric disorders.

One series of American reports dealt with the results of a study of 175 newly admitted men and women inpatients with families who were randomly assigned to three groups: standard inpatient care followed by outpatient care; brief admission to hospital followed by day care and discharge as outpatients; and brief admission to hospital followed only by discharge as outpatients. ${ }^{25-31}$ The design of the study was such that any patient readmitted during the two years after the index admission was again given the initially assigned treatment. Day patients were treated on the inpatient service, the main difference between them and inpatients being that they went home at night and weekends. Two thirds had a diagnosis of schizophrenia. Measurements of the patients' psychopathological features, how they functioned socially, the "family burden," and the costs of treatment were made at entry, after three months and six months, and at six monthly intervals up to two years after admission.

The average initial stay was 60 days for the standard group, and 11 days for both the brief groups. There was no difference in readmission rates, but the average number of days of inpatient care in the two years of the study did vary: 115 days for the standard group, 47 days for the group which received day care, and 27 days for the brief group which was merely discharged to the outpatient department after admission. The 